\title{
Extraskeletal osteosarcoma in right neck subcutaneous tissue: A case report of an extremely rare tumour
}

\author{
JIN-SHAN ZHANG ${ }^{1 *}$, GE WEN ${ }^{1 *}$, YUAN LIU ${ }^{2 *}$, ZHAO-HONG WU ${ }^{3 *}$, \\ GANG-DONG $\mathrm{CHEN}^{3}, \mathrm{HAO}_{\mathrm{WANG}}{ }^{1}$ and TONG-CHONG ZHOU${ }^{2}$ \\ ${ }^{1}$ Department of Radiation Oncology, Third Affiliated Hospital of Guangzhou Medical University; \\ ${ }^{2}$ Department of Radiation Oncology, Cancer Center of Guangzhou Medical University; ${ }^{3}$ Department of Thoracic Surgery, \\ Third Affiliated Hospital of Guangzhou Medical University, Guangzhou, Guangdong 510120, P.R. China
}

Received December 11, 2017; Accepted June 5, 2018

DOI: $10.3892 / \mathrm{mco} .2018 .1655$

\begin{abstract}
Extraskeletal osteosarcoma (ESOS) is a rare soft-tissue sarcoma that is treated with surgical resection, chemotherapy and radiotherapy; however, as it is now considered to be radiation resistance, it is associated with conflicting management principles and poor outcomes. A multimodality approach is currently used to treat ESOS, which entails the incorporation of multidrug chemotherapy and/or radiotherapy coupled with surgery to obtain the best outcome; however, there are many factors that influence the treatment effects and clinical outcomes of ESOS. In the present study, a case of an 81-year-old man who suffered from primary ESOS in the subcutaneous tissue of the right-hand side of the neck was reported. The patient was treated several times with partial resection and once with radiotherapy, and was still living following 3 years of follow-up. Thus, the present case report demonstrated that surgical resection and postoperative radiotherapy regimens may be favourable in the short term with a disease-free survival of $\sim 15$ months; however, patients are prone to relapse.
\end{abstract}

Correspondence to: Dr Tong-Chong Zhou, Department of Radiation Oncology, Cancer Center of Guangzhou Medical University, 78 Hengzhi Gang, Guangzhou, Guangdong 510120, P.R. China

E-mail: tongtongonggz@163.com

*Contributed equally

Abbreviations: ESOS, extraskeletal osteosarcoma; CT, computed tomography; CECT, contrast-enhanced CT; SMART, simultaneous modulated accelerated radiotherapy; RT, radiotherapy; RECIST 1.1, Response Evaluation Criteria In Solid Tumours version 1.1; SD, Stable disease; RTOG, Radiation Therapy Oncology Group; CEA, carcinoembryonic antigen; AFP, $\alpha$-fetoprotein; CTV, clinical target volume

Key words: osteosarcoma, subcutaneous tissue, extraskeletal, neck, radiation therapy, resection

\section{Introduction}

Extraskeletal osteosarcoma (ESOS) is a rare subtype of osteosarcoma, with which patients affected have distinct clinical features but similar prognostic factors and poor outcome compared to primary skeletal osteosarcoma (1), and its' management principles are not well defined and even conflicting, multimodality treatment remains standard (2-4). ESOS originating in the subcutaneous tissue is a rare occurrence, accounting for $<10 \%$ of all ESOS cases $(5,6)$. We present a case of ESOS primary in subcutaneous tissue of the right neck with which treated by both local resection and radiotherapy being relapsed several times (a brief summary of therapy on the patient see Table I).

\section{Case report}

Patient case. An 81-year-old man admitted to our hospital on May 26, 2014, presented with two weeks history of a mass founded on the right side of the neck, suffering with dull pain and swallowing; the patient provided written informed consent for participation in the present study. There was no history of any surgical procedure, trauma, local radiation exposure in head and neck, any long-term medication or addictions. There was no clinical evidence of metastatic disease. His family history and general physical examination was unremarkable. On physical examination, a hard-mobile subcutaneous mass approximately $4.0 \times 3.0 \mathrm{~cm}$ was palpable on the right side of the neck which moved with a slight tenderness. bilateral neck swollen lymph nodes were not touched. Karnofsky performance score (KPS) was 90. Blood count, serum alkaline phosphatase and tumor markers such as CEA and AFP were normal. CT (Fig. 1) scan revealed an uneven density soft tissue masses is of about $4.1 \times 3.0 \times 2.8 \mathrm{~cm}$ size in the rear of sternocleidomastoid on the right neck (Fig. 1A and C), contrast-enhanced CT (CECT) shows the mass peripherally enhanced with the necrosis in the center area, adjacent sternocleidomastoid and the right internal jugular vein were showed displaced (Fig. 1B and D).

First operation. A local widely resection was done on this patient in June 4, 2014. The operative procedure was performed under general anaesthesia, a subcutaneous mass with the size 


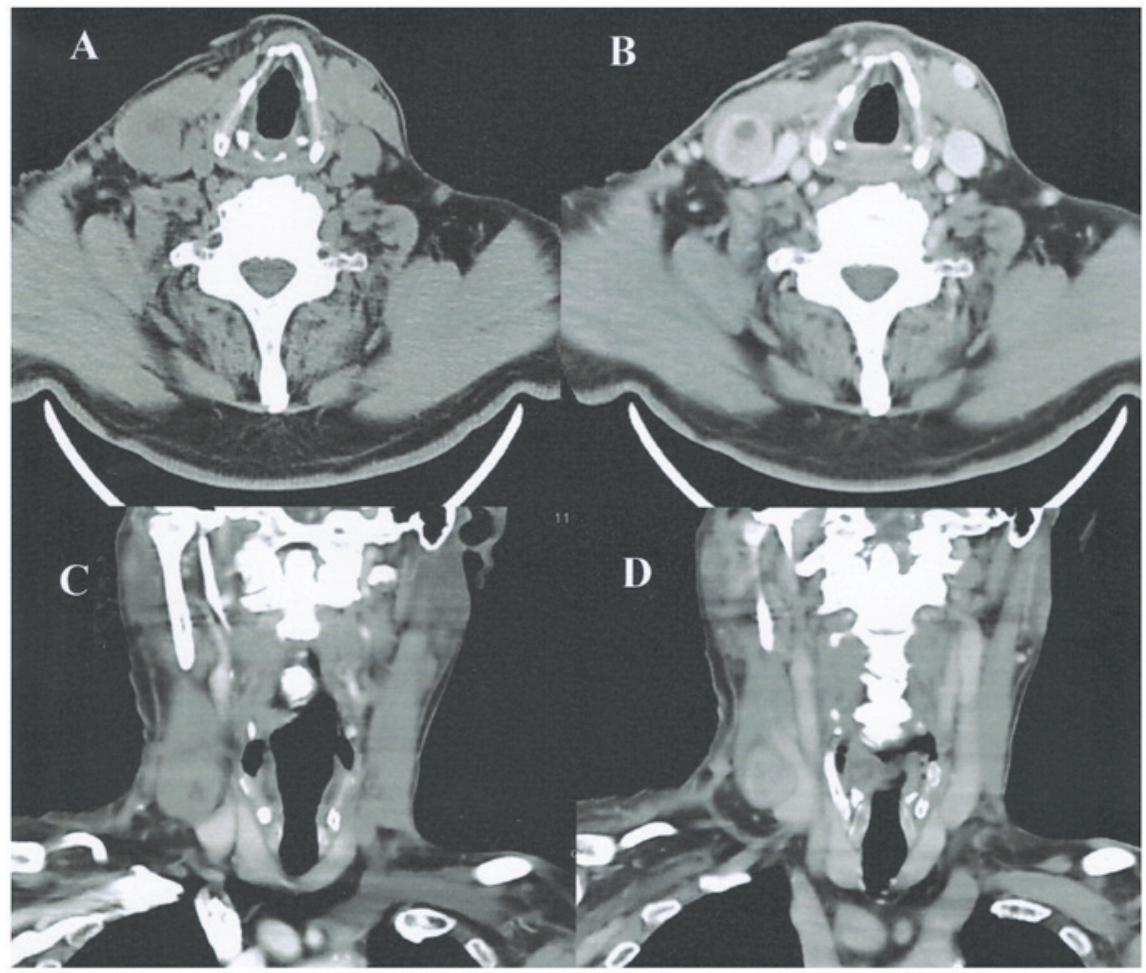

Figure 1. (A and C) CT scan showing an uneven density soft tissue masses in the rear of the sternocleidomastoid on the right neck. (B and D) Contrast-enhanced CT presenting the mass peripherally enhanced, the adjacent sternocleidomastoid and the right internal jugular vein, which were displaced. CT, computed tomography.

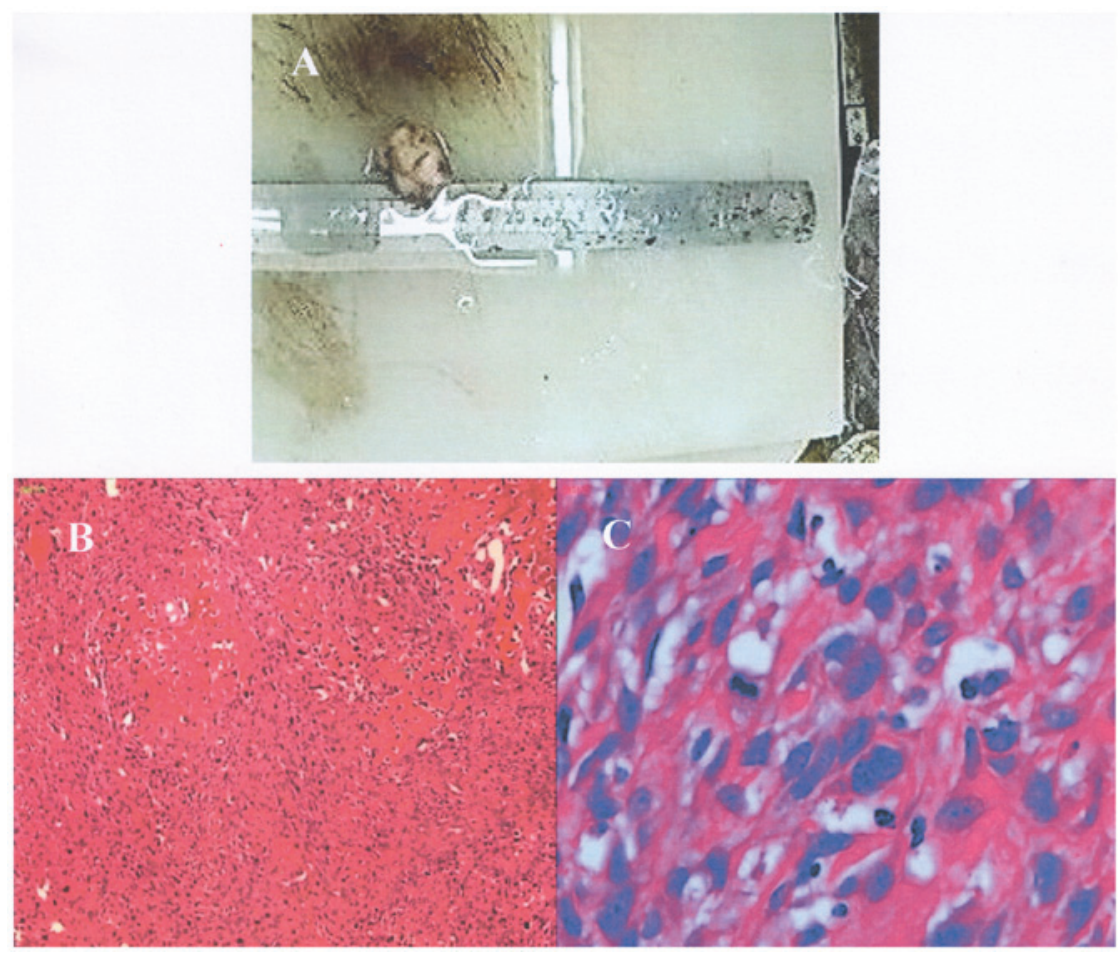

Figure 2. (A) A gray-white mass was resected, with a size of $\sim 4.0 \times 3.0 \times 3.0 \mathrm{~cm}$ (magnification, $\times 100$ ). (B and C) Microscopy of the resected tumour with hematoxylin and eosin staining revealed that the mass consisted of spindle-like, small round cells, with nuclei that were hyperchromatic and pleomorphic, and neoplastic cartilage formation (magnification, $\mathrm{x} 400$ ).

of about $4.0 \times 3.0 \times 3.0 \mathrm{~cm}$ was visible in the operation (Fig. 2A), and the tumour was incompletely removed as it closely related to the right jugular vein. On microscopy shows the resected mass consists of spindle, small round cells, with the nuclei hyperchromatic and pleomorphism, and neoplastic cartilage formation is seen (Fig. 2B and C), postoperative pathological 
diagnosis was as ESOS. The patient was unwilling to undergo chemotherapy or local radiotherapy after the operation.

Second operation and radiotherapy. Ten months later, On June 29,2015 , the patient was admitted to hospital again for 'postoperative and recurrence' of the right cervical osteosarcoma (Fig. 3A and B). A partial resection of the right neck mass was performed on July 3, 2015 (the second operation), and the postoperative pathology report indicated that the osteosarcoma of the right neck was recurred. Four weeks later, the patient underwent postoperative simultaneous modulated accelerated radiotherapy (SMART). The prescribed dose of CTV1 and CTV2 was $60.16 \mathrm{~Gy} / 32$ fractions and $70.08 \mathrm{~Gy} / 32$ fractions respectively, both once daily. 6 weeks after the radiotherapy, CT were performed to assess RT effects (Fig. 3C and D) was as stable disease (SD) according to the RECIST 1.1 criteria. During the period of RT, acute radioactive skin injury (level 2) occurred in the right neck skin (Fig. 3E and F) according to RTOG acute radiation injury classification criteria.

Third operation. The neck neoplasm to be founded increasing again from January 2017, with painful and obvious when swallowing, a mass was touched in the right neck, with mild tenderness and activity, CT showed recurrence (Fig. 4A and B). On February 20, 2017, the patient underwent the right neck neoplasm resection in condition of the resting compound anesthesia (the third operation). Postoperative pathological biopsy results showing (Fig. 4C and D): Neoplasm composed of spindle cells with nuclear atypia, nucleolus is clear, and multinucleated giant cells could be seen, nuclear fission is obvious, osteoid matrix is not obvious, and infringement of striated muscle tissue around them.

Fourth Operation and the follow-up. July 7, 2017, the patient underwent the partial resection for the mass enlarged once more with trachea compression, and damaged the skin. Partial mass with the range of about $6.0 \times 3.0 \times 3.0 \mathrm{~cm}$ was resected (Fig. 5). The patient is alive till now.

\section{Discussion}

Skeletal osteosarcoma (ESOS), also named as soft tissue osteosarcoma, is a rare malignant soft tissue sarcoma with histologic similarities to primary bone osteosarcoma but without attachment to the bone or periosteum, with malignant osteoid or bone (or both) formation and a uniform morphological sarcomatous tissue patterns (1). ESOS is a rare malignancy that the morbidity accounts for about 1 to $2 \%$ of all soft tissue sarcomas $(1,7)$, and less than $4 \%$ of all osteosarcomas $(7,8)$. Most of the ESOS occur in the upper and lower extremity, with the proportion of $\sim 40.0-67.5(1,9)$ to $\sim 85.7$ $-88.7 \%(4,10)$, but very few occur in the head and neck, with the constituent ratio is $2.5-3 \%(9,11)$ to $\sim 5-7.3 \%(1,3)$.These tumors generally arise with a high incidence of the median age is $~ 57-60.7$ years $(1,10)$, which usually located in the deep soft tissues without attachment to skeletal bones but were firmly attached to the fascia (12). This case of primary ESOS occurs in subcutaneous on the right side of the neck is exceedingly rare, which underwent four times of the operation, followed by once postoperative radiotherapy, but relapse for several times. 

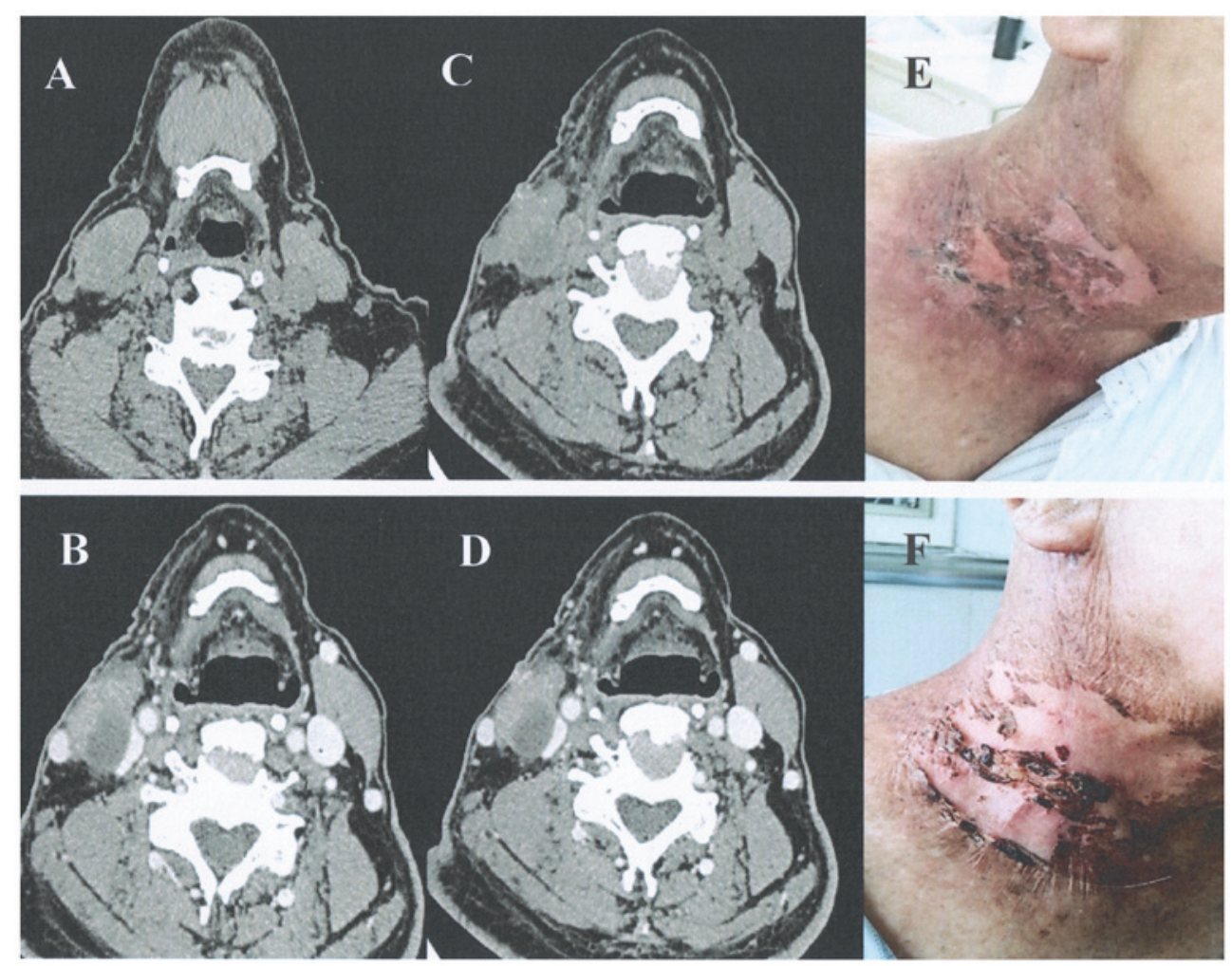

Figure 3. CT scan from June 29, 2015. (A) Unevenly distributed low density nodules were seen on the inside of the right sternocleidomastoid, with clear sternocleidomastoid boundaries, and flat compression of the right jugular vein. (B) Enhanced scanning: Arterial phase was slightly enhanced, and venous and delayed periods were further strengthened. (C) CT scan from November 10, 2015: Scan showing the medial low density nodules of the right sternocleidomastoid muscle were smaller than before and had decreased in density. (D) Enhanced scanning enhancement was not obvious. In terms of the Response Evaluation Criteria In Solid Tumors standard, it was 'Stable Disease'. (E) Acute radiation skin lesions (level 2) occurred in the right neck skin; however, (F) recovery was observed following local skin treatment. CT, computed tomography.

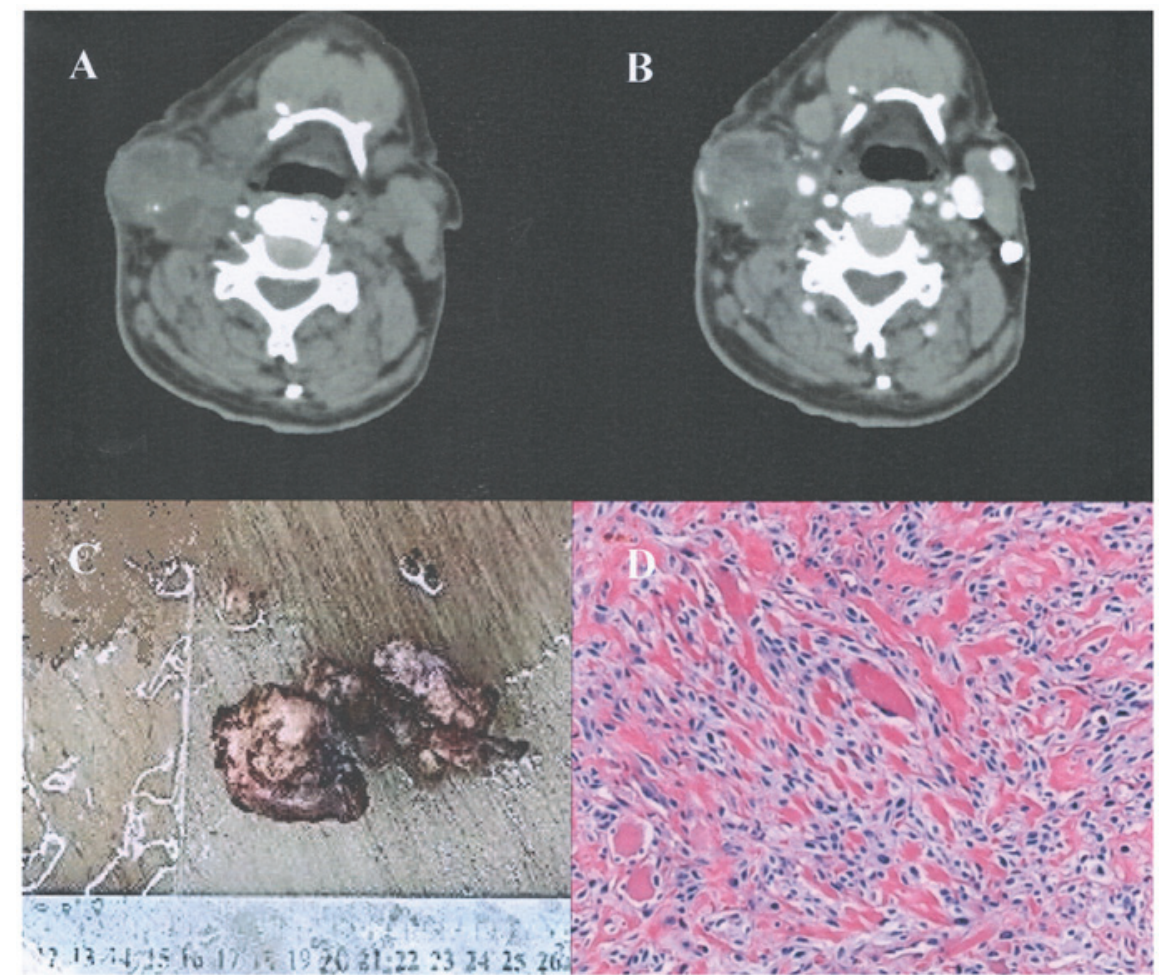

Figure 4. (A) CT scan showing an enlarged mass of $\sim 8.8 \times 6.0 \times 5.4 \mathrm{~cm}$, with $\mathrm{n}$ increased density on the right-hand side of the neck. (B) Contrast-enhanced CT revealed the mass was enhanced in homogeneity, with a fuzzy surrounding fat gap, adjacent skin thickening and a moderately enhanced nature. (C) Surgical excision contains multiple tissue fragments. (D) Hematoxylin and eosin staining (magnification, x200). The tumor was composed of spindle cells, nuclear heteromorphism, nucleolus clarity, multiple nuclear giant cells, nuclear fission, the osteoid matrix was not obvious, and the surrounding rhabdomyosis was infringed. CT, computed tomography. 

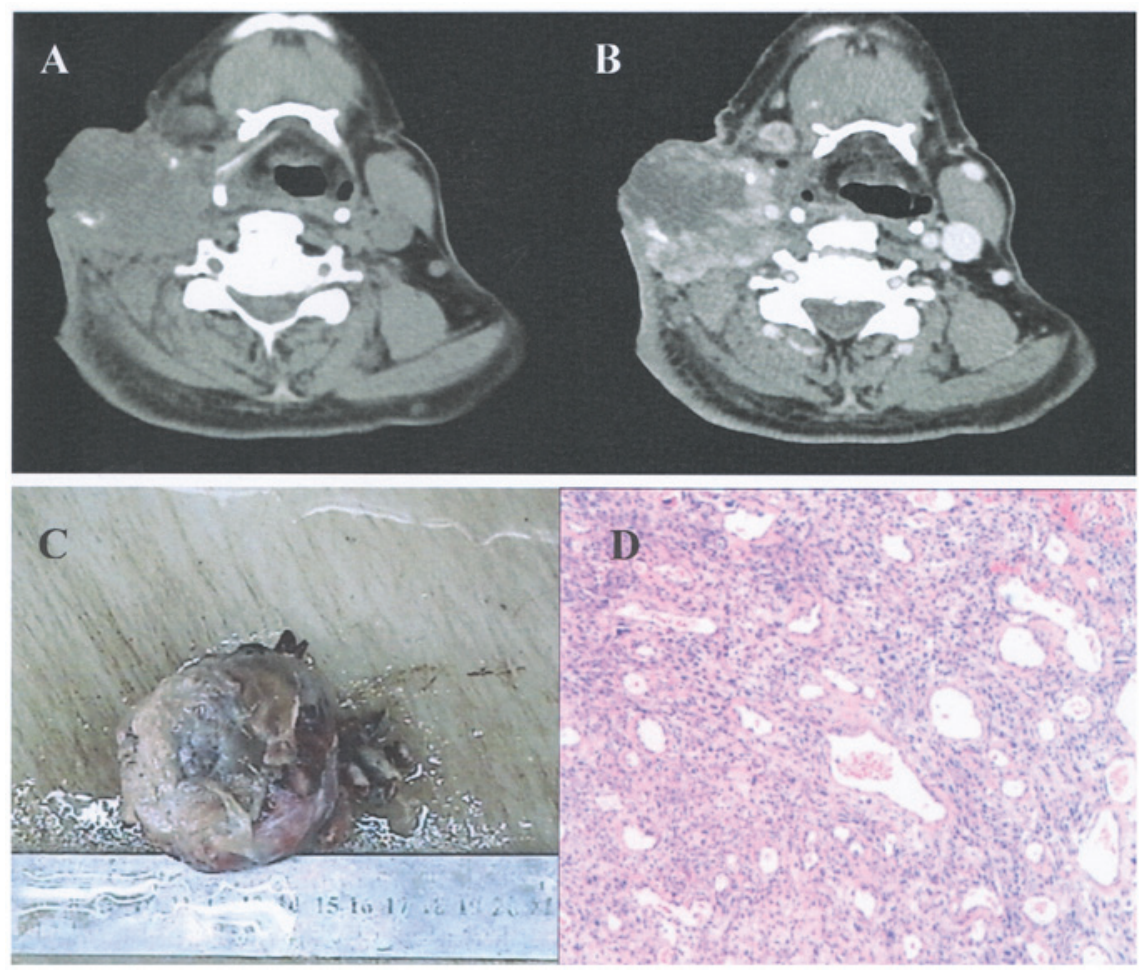

Figure 5. (A) CT scan showing large clumpy, low density soft tissue masses on the right-hand side of neck with the range of $\sim 9.2 \times 6.6 \times 5.3 \mathrm{~cm}$, with irregular speckle calcification on the inside of the mass; the mass was not clear with the sternocleidomastoid, and the right pharyngeal space disappeared. (B) Contrast-enhanced CT showing an inhomogeneous peripheral enhancement; no enhanced necrotic areas were seen in the center and the total vein of the right neck and the internal jugular vein were compressed. (C) The resected mass had a cauliflower-like, hard texture, and was (D) confirmed as relapsed extraskeletal osteosarcoma by pathology (Hemaxtoylin and eosin; magnification, x100). CT, computed tomography.

ESOS patients starting symptoms tend to be progressive enlargement of tumors and rarely causing pain or tenderness, the preoperative duration of symptoms ranged from 2 weeks to 25 years (median, 6 months) (12). But ESOS could firstly manifest as spontaneous tumor lysis syndrome which represents an oncological emergency must be treated as soon as possible (13). ESOS is a rare mesenchymal malignancy of soft tissue, histologically indistinguishable from primary osteosarcoma of bone. However, there are distinct differences in demographics, imaging features, prognosis, and management compared with osteogenic osteosarcoma (8), its correct diagnosis may depend on a combination of clinical, radiographic, and pathologic findings $(8,12,14-16)$.

There are several treatment methods for ESOS, including surgery, radiation therapy, and chemotherapy $(2-4,10,11)$, surgical resection is dominated, 85 to $98 \%(10,11)$, even all patients with localized disease were managed with surgical resection of the primary tumor (4), radical resections are effective for local control and have the best chance of cure for ESOS (8), 5-year OS and 5-year disease-free survival (DFS) was 51.4 and $43 \%$ respectively (10), but the proportion of patients treated alone with surgery was low (21.8\%) (10), and its effect on distant metastasis is not so clear, multiagent chemotherapy may be help to reduce distant metastasis, a trend towards increased length of survival was found in patients who received chemotherapy compared to those who did not (16.4 months vs. 9.3 months) (2), the gemcitabine-docetaxel chemotherapy regimen was considered as well-tolerated and induced a long lasting partial response for $\sim 14$ months in the treatment of ESOS (17). Higher survival was observed in patients who received perioperative chemotherapy with a trend in favour of multiagent osteosarcoma-type regimen which included doxorubicin, ifosfamide and cisplatin (10), and postoperative adjuvant external beam radiotherapy being considered to improve local control rate and preserve organ function $(18,19)$, and especially the patients who with tumour $>5 \mathrm{~cm}$ and R0 margins seems to benefit more from RT (10), and there is a tendency to extend 5-y DFS in patients who underwent postoperative adjuvant RT compared with surgery alone (66 vs. $42 \%, \mathrm{P}=0.38$ ) (11), though RT was not associated with a lower disease-related mortality rate or a longer event-free survival (4). However, the available data are contradictory with regard to the use of chemotherapy and radiotherapy regimens in the management of ESOS, radiographic response rates and pathologic complete response rates to doxorubicin-based systemic therapy are low (3), no significant association of disease-specific or event-free survival was found with the addition of radiation, chemotherapy, or both to surgery, radiation and chemotherapeutic treatment were not associated with a lower incidence of death due to disease or a longer event-free survival (4). Currently, a multimodality approach is used to treat extra skeletal osteosarcoma, which entails incorporation of multidrug chemotherapy and /or radiotherapy along with the surgery to get the best outcome in terms of disease specific survival and local relapse free survival (10). In fact, there are many factors influence the treatment effects and clinical outcomes of ESOS, including tumor size, tumor location, pathology classification and grade, clinical stage, surgical margin, choice of chemotherapy drugs, age $(1,4,7,9,11,20)$, expression of oncogenes $(21,22)$, and so on. 
In this case of ESOS, lesion located in subcutaneous on the right side of the neck, in the posterior of the sternocleidomastoid, with the adjacent sternocleidomastoid and the right internal jugular vein were displaced, thus, the tumour was incompletely removed. The postoperative chemotherapy and local radiotherapy seem to be necessary $(2,10,11,16,17)$, but the patient refused the postoperative radiation and chemotherapy, with the result of the local recurrence after about nine months of the first operation. In the case, the patient voluntarily received local radiotherapy after the second operation, and DFS is about 15 months. Third resection performed when relapsed again, second course of radiotherapy was not considered for having had a history of local radiotherapy and an obvious skin injury, but the patients still refused to chemotherapy, 5 months later, the patient underwent another operation for relapsed once more.

\section{Acknowledgements}

Not applicable.

\section{Funding}

No funding was received.

\section{Availability of data and materials}

The datasets used and/or analyzed during the current study are available from the corresponding author on reasonable request.

\section{Authors' contributions}

JSZ participated in the conception and design of the case report and wrote the manuscript. GW and YL evaluated the patient, participated in the radiotherapy procedures and were major contributors in writing the manuscript. $\mathrm{ZHW}$ performed the surgical management procedures and reviewed the manuscript. GDC conducted the surgical management procedures. HW participated in radiotherapy and wrote the manuscript. TCZ assisted with the treatment of radiotherapy and reviewed the manuscript. All authors have read and approved the final draft.

\section{Ethics approval and consent to participate}

The patient provided written informed consent for participation in the present study.

\section{Consent for publication}

The patient provided written informed consent for the publication of any associated data and images in this case report.

\section{Competing interests}

The authors declare that they have no competing interests.

\section{References}

1. Thampi S, Matthay KK, Boscardin WJ, Goldsby R and DuBois SG: Clinical features and outcomes differ between skeletal and extraskeletal osteosarcoma. Sarcoma 2014: 902620, 2014.
2. Nystrom LM, Reimer NB, Reith JD, Scarborough MT and Gibbs CP Jr: The treatment and outcomes of extraskeletal osteosarcoma: Institutional experience and review of the literature. Iowa Orthop J 36: 98-103, 2016.

3. Ahmad SA, Patel SR, Ballo MT, Baker TP, Yasko AW, Wang X, Feig BW, Hunt KK, Lin PP, Weber KL, et al: Extrasseous osteosarcoma: Response to treatment and long-term outcome. J Clin Oncol 20: 521-527, 2002.

4. Choi LE, Healey JH, Kuk D and Bennan MF: Analysis of outcomes in extraskeletal osteosarcoma: A review of fifty-three cases. J Bone Joint Surg Am 96: e2, 2014.

5. Healy C, Kahn BL and Kenan S: Subcutaneous extraskeletal osteosarcoma of the forearm: A case report and review of the literature. Skeletal Radiol 45: 1307-1311, 2016.

6. Nakamura T, Matsumine A, Nishimura K, Yokoyama H, Murata T, Uchida A and Sudo A: Extraskeletal subcutaneous osteosarcoma of the upper arm: A case report. Oncol Lett 2: 75-77, 2011.

7. Bane BL, Evans HL, Ro JY, Carrasco CH, Grignon DJ, Benjamin RS and Ayala AG: Extraskeletal osteosarcoma: A clinicopathologic review of 26 cases. Cancer 65: 2762-2770, 1990.

8. Mc Auley G, Jagannathan J, O'Regan K, Krajewski KM, Hornick JL, Butrynski J and Ramaiya N: Extraskeletal osteosarcoma: Spectrum of imaging findings. AJR Am J Roentgenol 198: W31-W37, 2012.

9. Lee JY, Fefsch JF, Wasdhal DA, Lee BP, Pritchard DJ and Nascimento AG: A review of 40 patients With extraskeletal osteosarcom. Cancer 76: 2253-2259, 1995.

10. Longhi A, Bielack SS, Grimer R, Whelan J, Windhager R, Leithner A, Gronchi A, Biau D, Jutte P, Krieg AH, et al: Extraskeletal osteosarcoma: A European musculoskeletal oncology society study on 266 patients. Eur J Cancer 74: 9-16, 2017.

11. Sio TT, Vu CC, Sohawon S, Van Houtte P, Thariat J, Novotny PJ, Miller RC and Bar-Sela G: Extraskeletal osteosarcoma: An international rare cancer network study. Am J Clin Oncol 39: 32-36, 2016.

12. Chung EB and Enzinger FM: Extraskeletal osteosarcoma. Cancer 60: 1132-1142, 1987.

13. Catania VE, Vecchio M, Malaguarnera M, Madeddu R, Malaguarnera G and Latteri S: Tumor lysis syndrome in an extraskeletal osteosarcoma: A case report and review of the literature. J Med Case Rep 11: 79, 2017.

14. Fanburg-Smith JC, Bratthauer GL and Miettinen M: Osteocalcin and osteonectin immunoreactivity in extraskeletal osteosarcoma: A study of 28 cases. Hum Pathol 30: 32-38, 1999.

15. Secil M, Mungan U, Yorukoglu K and Dicle O: Case 89: Retroperitoneal extraskeletal osteosarcoma. Radiology 237: 880-883, 2005.

16. Hu B, Liu Y, Cheng L, Li W and Cao X: SPECT/CT imaging of retroperitoneal extraskeletal osteosarcoma. Clin Nucl Med 39: 200-202, 2014.

17. Strippoli S, Traversa M, Cramarossa A, Popescu O, Lorusso V and Guida M: Long-term response of gemcitabine plus docetaxel chemotherapy regimen for extraskeletal osteosarcoma: A case report. Oncol Lett 9: 2567-2571, 2015.

18. Bhatt NR, Kakked GA, Merchant R and Bhatt R: Extraskeletal osteosarcoma of the larynx: An extremely unusual tumour. BMJ Case Rep 2014: pii: bcr2014206759, 2014.

19. Casey DL, van de Rijn M, Riley G, Tung KW, Mohler DG and Donaldson SS: Extraskeletal osteosarcoma of the hand: The role of marginal excision and adjuvant radiation therapy. Hand (NY) 10: 602-606, 2015.

20. Abramovici LC, Hytiroglou P, Klein RM, Karkavelas G, Drevelegas A, Panousi E and Steiner GC: Well-differentiated extraskeletal osteosarcoma: report of 2 cases, 1 with dedifferentiation. Hum Pathol 36: 439-443, 2005.

21. Jour G, Wang L, Middha S, Zehir A, Chen W, Sadowska J, Healey J, Agaram NP, Choi L, Nafa K and Hameed M: The molecular landscape of extraskeletal osteosarcoma: A clinicopathological and molecular biomarker study. J Pathol Clin Res 2: 9-20, 2015.

22. Yamashita K, Kohashi K, Yamada Y, Nishida Y, Urakawa H, Oda Y and Toyokuni S: Primary extraskeletal osteosarcoma: A clinicopathological study of 18 cases focusing on MDM2 amplification status. Hum Pathol 63: 63-69, 2017. 